\title{
The early effect of dapagliflozin on strain and tissue Doppler parameters of diastolic function in diabetic patients with heart failure with reduced ejection fraction
}

Spyridon Maragkoudakis ${ }^{1}$, Maria Marketou², Vasiliki Katsi ${ }^{3}$, Alexandros Patrianakos ${ }^{2}$, Labrini Tsigkriki ${ }^{1}$, Maria Mamaloukaki ${ }^{1}$, Kostas Tsioufis ${ }^{3}$, George Kochiadakis ${ }^{2}$, Fragkiskos Parthenakis ${ }^{2}$

${ }^{1}$ Department of Cardiology, General Hospital Saint George of Chania, Chania, Greece ${ }^{2}$ Department of Cardiology ,University Hospital of Heraklion, Heraklion Greece ${ }^{3}$ Department of Cardiology, Hippokrateion University Hospital, Athens, Greece

Submitted: 4 July 2021

Accepted: 4 August 2021

Arch Med Sci Atheroscler Dis 2021; 6: e176-e181

DOI: https://doi.org/10.5114/amsad.2021.109685

Copyright (c) 2021 Termedia \& Banach

\section{Abstract}

Introduction: Heart failure (HF) with reduced ejection fraction (HFrEF) remains a challenging problem due to its high mortality rate. The PARADIGM $\mathrm{HF}$ trial and a new class of drugs - angiotensin receptor-neprilysin inhibitors (ARNIs) - managed to change the current perception of HF treatment by reducing cardiovascular mortality and morbidity as well as HF hospitalizations compared with enalapril and have emerged as an evidence-based therapy for HFrEF. Another novelty in HF therapy is dapagliflozin, a sodium-glucose transporter-2 inhibitor (SGLT2i) which decreased the rates of cardiac death and worsening of HF in the DAPA-HF trial, when added in other guideline recommended therapy. A recent study evaluated the potency of dapagliflozin in terms of mortality and deterioration of HF, in patients taking sacubitril/ valsartan and in patients who were naive.

Material and methods: A prospective cohort study of 30 symptomatic HF patients with $\mathrm{EF}<35 \%$ (aged $65 \pm 10$ years) was conducted. Diabetic (2TDM) patients of NYHA status II-III, previously treated with ARNI, $\beta$-blocker, and mineralocorticoid receptor antagonists (MRA) were included. Dapagliflozin was added to their therapy.

Results: Echocardiographic evaluation revealed improvement of both conventional tissue Doppler and diastolic strain parameters by dapagliflozin addition on HF therapy.

Conclusions: Dapagliflozin impact on diastolic function may explain the symptom amelioration and the improvement of quality of life. And more specifically, the ratio of early diastolic transmitral flow velocity to global strain rate at the early filling phase of diastole (E/SRE)may be considered a reliable index of $\mathrm{HF}$ therapy responders.

Key words: dapagliflozin, strain and tissue Doppler parameters, diastolic function.

\section{Introduction}

In heart failure (HF) with reduced ejection fraction (HFrEF) patients the most common outcomes in clinical trials are cardiovascular death and hospitalization rate. In the past years a great effort has been made and new horizons have been inaugurated with the introduction of sodium-glucose transporter-2 inhibitor (SGLT2i) and angiotensin receptor-
Corresponding author: Spyridon Maragkoudakis MD Department of Cardiology General Hospital Saint George of Chania Chania, Greece E-mail: smaragoudakis79@ hotmail.com 
neprilysin inhibitors (ARNIs) in the battle against heart failure.

First of all, the PARADIGM HF trial showed that sacubitril/valsartan is more efficient in the reduction of cardiovascular (CV) death and HF hospitalization than enalapril [1-6] and the European Society of Cardiology upgraded the level of recommendation of sacubitril/valsartan in symptomatic patients with left ventricle ejection fraction (LVEF) $\leq 35 \%$ under angiotensin converting enzyme inhibitor, $\beta$-blocker, and MRA treatment into class I, level of evidence $B$ [7].

Dapagliflozin managed to reduce $C V$ death and deterioration of HFrEF patients in the DAPA-HF trial, which was considered a breakthrough in the spectrum of HF. Apart from the primary composite end point, the Kansas City Cardiomyopathy Questionnaire Score (KCCQ) was used to study the impact of SGLT2i on symptoms, physical function and quality of life. The addition of dapagliflozin in HFrEF patients' treatment not only improved their health status but also significantly fewer patients experienced clinically deterioration and required intensification of therapy [8].

We hypothesized that this beneficial action of SGLT2i may be attributed partly to their effect on diastolic function.

\section{Material and methods}

A prospective cohort study of 30 symptomatic $\mathrm{HF}$ patients with $\mathrm{EF}<35 \%$ (aged $65 \pm 10$ years) was conducted. Diabetic (type 2 diabetes mellitus - 2TDM) patients of NYHA status II-III, previously treated with ARNI, $\beta$-blocker, and mineralocorticoid receptor antagonists (MRA) were included. According to our eligible criteria our patients needed to be stable without intensification of their therapy in the last 4 weeks before enrollment. Clinical examination and complete echocardiographic evaluation were performed at baseline and 30 days after the addition of SGLT2i to their treatment. All participants provided written informed consent. The study protocol complies with the ethical guidelines of the 1975 Declaration of Helsinki.

\section{Echocardiographic evaluation}

2D echocardiography and Doppler measurements were used for the evaluation of left ventricular function, and more specifically, peak early (E-wave) and late (A-wave) transmitral filling velocities and their ratio $E / A$, deceleration time of $E$ velocity (DTE) and the mitral inflow velocity $E$ to tissue Doppler E' (E/E') were measured. Two-dimensional speckle-tracking echocardiography (STE) with frame rates of $50-85 \mathrm{~Hz}$ was used for the assessment of peak early and late diastolic rate (SRE and SRL). All apical views were used and their average values were regarded as the global SRE and SRL.

\section{Statistical analysis}

Mean \pm standard deviation (SD) was used for continuous variables whereas for categorical variables frequency (\%) was used. The paired samples $t$-test or paired Wilcoxon test was used for the evaluation of dapagliflozin addition to the HF treatment.

\section{Results}

Thirty symptomatic patients with mean age $62 \pm 9$ years ( 20 males and 10 females) were enrolled in our study; $60 \%$ of them described NYHA II symptoms and 40\% NYHA III, and both groups were under valsartan/sacubitril, $\beta$-blocker, MRA therapy. Dapagliflozin $10 \mathrm{mg}$ was added as an adjunct therapy for 2TDM but also as a novel therapy of HF in diabetic patients. The characteristics of the enrolled patients are listed in Table I.

After 30 days of therapy with dapagliflozin, blood pressure was reduced significantly and the echocardiographic parameters of diastolic function were improved. More specifically, DTE, E/A, E/E' were ameliorated (7.5 $\pm 1.50,-0.300 \pm 0.0316$,

Table I. Baseline characteristics and demographics of the enrolled patients $(n=30)$

\begin{tabular}{|c|c|}
\hline Characteristics & Results \\
\hline Age & $65 \pm 10$ \\
\hline Males/females, $n(\%)$ & $20 / 10(66 / 34 \%)$ \\
\hline \multicolumn{2}{|l|}{ NYHA class, $n(\%)$ : } \\
\hline NYHA II & $18(60)$ \\
\hline NYHA IV & $12(40)$ \\
\hline Ischemic cardiomyopathy, $n$ (\%) & $20(67)$ \\
\hline LVEF (\%) & $32.1 \pm 2.5$ \\
\hline Diabetes, $n(\%)$ & $30(100)$ \\
\hline Hypertension, $n$ (\%) & $20(67)$ \\
\hline Atrial fibrillation, $n(\%)$ & $10(33)$ \\
\hline \multicolumn{2}{|l|}{ Treatment, $n(\%)$ : } \\
\hline ARNIS & $30(100)$ \\
\hline Beta-blockers & $30(100)$ \\
\hline MRAs & $30(100)$ \\
\hline Ivabradine & $8(29)$ \\
\hline Digoxin & $10(33)$ \\
\hline Antidiabetic treatment & $30(100)$ \\
\hline Antiplatelets & $20(67)$ \\
\hline Anticoagulants & $10(33)$ \\
\hline$I C D$ & $24(80)$ \\
\hline CRT & $5(17)$ \\
\hline Diuretics & $30(100)$ \\
\hline
\end{tabular}


Spyridon Maragkoudakis, Maria Marketou, Vasiliki Katsi, Alexandros Patrianakos, Labrini Tsigkriki, Maria Mamaloukaki, Kostas Tsioufis, George Kochiadakis, Fragkiskos Parthenakis

Table II. Systolic and diastolic blood pressure as well as echocardiographic parameters at baseline and after 1-month therapy with dapagliflozin

\begin{tabular}{|lcccc|}
\hline Parameters & Baseline & 30 days of therapy & Mean difference & $P$-value \\
\hline Systolic BP & $121 \pm 9.16$ & $114 \pm 9.60$ & $-7.00 \pm 0.648$ & $<0.001$ \\
\hline Diastolic BP & $65.4 \pm 11.6$ & $59.6 \pm 10.3$ & $-5.50 \pm 0.667$ & $<0.001$ \\
\hline DTE $[\mathrm{ms}]$ & $124 \pm 9.61$ & $130 \pm 10.35$ & $7.5 \pm 1.50$ & $<0.001$ \\
\hline E/A & $1.55 \pm 0.237$ & $1.29 \pm 0.156$ & $-0.300 \pm 0.0316$ & $<0.001$ \\
\hline E/E' & $12.4 \pm 1.66$ & $10.4 \pm 1.33$ & $-1.97 \pm 0.176$ & $<0.001$ \\
\hline SRE $\left[s^{-1}\right]$ & $0.698 \pm 0.0894$ & $0.775 \pm 0.0894$ & $0.0850 \pm 0.00867$ & $<0.001$ \\
\hline E/SRE $[\mathrm{cm}]$ & $255.0 \pm 22.9$ & $210.0 \pm 24.9$ & $-44.0 \pm 3.35$ & $<0.001$ \\
\hline SRE/SRL & $0.824 \pm 0.0645$ & $1.033 \pm 0.1302$ & $0.200 \pm 0.0189$ & $<0.001$ \\
\hline
\end{tabular}

$A$ - late diastolic mitral flow velocity, BP - blood pressure, DTE - deceleration time of $E$, $E$ - peak early diastolic mitral flow velocity, $E^{\prime}$ - mitral annulus early diastolic velocity, SRE - global peak early diastolic rate, SRL - global peak late diastolic rate.

$-1.97 \pm 0.176$ respectively). Diastolic strain indexes were also influenced by dapagliflozin administration, SRE (0.0850 \pm 0.00867$)$, E/SRE $(-44.0 \pm 3.35)$ and SRE/SRL (0.200 \pm 0.0189$)$. Among these indexes E/E', E/SRE and SRE/SRL responded the most to dapagliflozin treatment with an effect size Cohen's $d$ of $-2.04,-2.48$ and 2.03 respectively. Our results are presented in Table II.

\section{Discussion}

Our data support the evidence that SGLT2i ameliorate both the conventional echocardiographic indexes and strain parameters of diastolic function and this effect is noted quite promptly. The ratio of early diastolic transmitral flow velocity to global strain rate at the early filling phase of diastole (E/SRE) seems to correlate well with LV filling pressures, and may be used as a prognostic tool in patients with HFrEF [9-11]. We have to emphasize that the diastolic strain improvement was observed 30 days after the initiation of dapagliflozin treatment, and thus it may be considered an early marker of improvement and adequacy of treatment apart from BNP.

The myocardial dysfunction observed in 2TDM is a process occurring progressively. Initially, we have an early phase without symptoms during which the left ventricle undergoes hypertrophy and diastolic dysfunction develops and then the late stage during which alterations in microcirculation take place, left ventricular size increases and myocardial function is impaired [12]. The course of alterations in myocardial performance is influenced by several factors including hypertension, coronary artery disease, obesity, and age [13].

In heart failure with preserved (HFpEF) and mid-range ejection fraction (HFmEF) the earliest abnormality detected is LV diastolic impairment [14], which is associated with adverse outcomes in HF patients $[15,16]$. Several studies of DM patients are in accordance and indicate that the initial alteration in the course of diabetic cardio- myopathy is diastolic dysfunction. Ernande et al. reported that $47 \%$ of asymptomatic patients with 2TDM and preserved LVEF under adequate blood pressure control had impaired diastolic function [17]. Consequently in HF and DM patients we should not focus only on systolic function and ejection fraction; the course of the disease starts from earlier signs of impairment such as diastolic dysfunction.

Thus, we need feasible parameters for the early detection of the aforementioned changes which will also delineate other clinical characteristics of our patients such as symptoms and the ability to exercise. These may be the echocardiographic indexes of diastolic function and the global strain and, as Oikonomou et al. demonstrated, both of them correlate well with the exercise capacity, quality of life and physical status of HF patients $[17,18]$. Moreover, DM that is not well controlled causes not only early diastolic but also systolic dysfunction, reduced GLS values can coexist with impaired diastolic echocardiographic parameters and, more specifically, patients with GLS $<18.9 \%$ had worse outcome $[19,20]$. Thus, the use of feasible parameters for the early detection of the aforementioned alterations seems to be mandatory.

The EMPA-REG OUTCOME trial investigated the effects of SGLT2i (empagliflozin), compared with placebo, and showed reduction of cardiovascular mortality and rehospitalizations for HF. Thus the burden of cardiovascular mortality and morbidity is reduced but their impact on diastolic function remains terra incognita [21]

Dapagliflozin is an effective antidiabetic agent reducing as monotherapy glycosylated hemoglobin $(-0.68 \%$ with $1 \mathrm{mg},-0.72 \%$ with $2.5 \mathrm{mg}$, $-0.82 \%$ with $5 \mathrm{mg}$ ) compared with placebo ( $+0.02 \%$, $p<0.0001)$; further reduction is achieved in combination with metformin (-0.67\% with $2.5 \mathrm{mg}$, $-0.70 \%$ with $5 \mathrm{mg}$ and $-0.84 \%$ with $10 \mathrm{mg}$ ) compared with placebo $(-0.30 \%$, all $p<0.001)$. Dapagliflozin seems to result in lower rates of withdrawal as well as in lower incidence of addition 
of rescue medication [22]. In naïve patients with glycated hemoglobin $\left(\mathrm{HbA}_{1 \mathrm{c}}\right)$ levels of $9 \%$, the reduction of $\mathrm{HbA}_{1 \mathrm{c}}$ levels was comparable to the one achieved by metformin-XR ((-1.45\%)-1.44\% respectively) [23]. Dapagliflozin has been studied extensively as an adjunct therapy. A meta-analysis of trials including dapagliflozin $10 \mathrm{mg}$ as part of dual or triple antidiabetic treatment showed that the net $\mathrm{HbA}_{1 \mathrm{c}}$ reduction achieved was $-0.54 \%$ [24]. Tight glycemic control and lower levels of $\mathrm{HbA}_{1 \mathrm{c}}$ will enhance diastolic function.

The DECLARE-TIMI 58 trial, which included 17160 patients, revealed that dapagliflozin reduced CV death (CVD) and HF hospitalizations by $4.9 \%$ and $5.8 \%$ respectively and also showed non-inferiority versus placebo concerning major cardiac adverse events [25]. This cardioprotective effect of dapagliflozin is probably due to glycemic control as already noted, but also due to the blood pressure, plasma volume, and arterial stiffness reduction [26]. Additionally, two other mechanisms are implicated in the cardioprotective role of the drug. It seems that ketone body formation and oxidation due to SGLT2i treatment have antiarrhythmic activity and, via inhibiting the myocardial $\mathrm{Na}^{+} / \mathrm{H}^{+}$exchanger, it reduces the intracellular $\mathrm{Na}^{+}$ concentration, which in turns leads to secondary reduction of intracellular and mitochondrial concentration [26].

SGLT2i are associated with lower blood pressure, and actually it seems that these agents may cause a mean reduction of $3-5 \mathrm{~mm} \mathrm{Hg}$ when used as monotherapy [27]. This effect is mainly attributed to the osmotic diuresis of dapagliflozin. SGLT2i act on the proximal convoluted tubule, inhibit glucose reabsorption and enhance its excretion followed by natriuresis [28].

Furthermore, dapagliflozin augments natriuresis in conjugation with loop diuretics by $36 \%$ due to enhanced delivery of $\mathrm{NaCl}$ to the loop of Henle. Thiazides and SGLT2i may also act synergistically, and their effect seems to be beneficial in heart failure. The reduction of systolic blood pressure is not followed by an increase of the heart rate, and it seems that reflex activity of the sympathetic nervous system (SNS) is not stimulated. SGLT2i via an unclear mechanism correct overactivity of the SNS during sleep and restore a normal circadian BP rhythm [29]. Solomon et al. reported that amelioration of diastolic performance depends on tight systolic blood pressure control [30]. Actually, among 228 patients, more prominent enhancement of diastolic function was noted in those who achieved lower levels of systolic blood pressure [30].

Obesity is another well-known cause of diastolic function impairment. Abdominal visceral adipose tissue measured by computed tomography seems to be well correlated with echocardiographic parameters of diastolic function in asymp- tomatic patients with $\mathrm{DM}[31,32]$. Urinary glucose excretion mediated by SGLT2i will be followed by calorie loss and thus most of the studies have exhibited 2-3 kg weight loss in patients with DM. Though it was thought that this weight loss was the result of osmotic diuresis, it was elucidated that it was accompanied by a reduction in body fat mass [33, 34].

Thus, SGLT2 $\mathrm{i}$ via the reduction in blood pressure, $\mathrm{HbA}_{1 \mathrm{c}}$ levels and weight loss changes seem to exert a significant impact on LV function, and ameliorate the diastolic function indexes. More specifically, dapagliflozin according to our findings improves diastolic function and is implicated in regression of the restrictive filling pattern.

In accordance with our results, Verma et al. reported that the administration of $10 \mathrm{mg}$ of empagliflozin in patients with T2DM and cardiovascular disease reduced both left ventricular mass index (LVMI) and e' as diastolic echocardiographic parameter [35]. Matsutani et al. evaluated the effect of canagliflozin on diastolic function and found that both LVMI and E/e' were improved. Our study, apart from the conventional indexes of diastolic function, used E/SRE ratio as a more accurate measurement of diastolic function. Dapagliflozin managed to decrease E/E' ratio, which is a marker of ventricular filling pressures as well as diastolic strain parameters. The E/SRE ratio is considered to be a more precise marker than E/E' for LV filling pressures as well as an accurate predictor of prognosis of patients with HFrEF [36-38].

Furthermore, our study assessed the co-administration impact of valsartan/sacubitril and dapagliflozin on diastolic function. A recent study in DAPA HF patients investigated the impact of dapagliflozin on CVD primary outcome and on episodes of HF hospitalization in 2 groups of patients, those who were taking sacubitril valsartan and those who were naïve to ARNIs. Dapagliflozin was similarly efficacious in both groups, and the rate of adverse effects due to volume depletion was similar in both groups [4]. Although we could expect some degree of overlap in their mechanism of action and attenuation of therapy, the lack of interaction and therapy withdrawal suggests a distinct cardioprotective mechanism [4]. In our study patients tolerated well the co-administration of both agents and the amelioration of symptoms is consistent with the improvement of echocardiographic indexes of diastolic function.

Our study focused on the impact of valsartan/ sacubitril and dapagliflozin on diastolic function and supports the pivotal role of 2D-STE for the evaluation of HF patients.

The relatively small sample size is a limitation of our study. However, our results are clear and can be used for conducting larger trials concern- 
ing the novel therapeutic agents of HF valsartan/ sacubitril and dapagliflozin.

In conclusion, in this pilot study classical diastolic echocardiographic parameters as well as diastolic strain parameters were improved promptly after initiation of dapagliflozin therapy. The ratio of early diastolic transmitral flow velocity to global strain rate at the early filling phase of diastole (E/ SRE) may be considered a reliable index of HF therapy responders. Nevertheless, a larger sample of patients will elucidate the exact pathophysiological impact of dapagliflozin on HFrEF patients' diastolic function. Our results are consistent with the results of larger trials that suggest that the use of ARNIs and SGLT2i in HFrEF seems to be beneficial for morbidity, mortality and quality of life.

\section{Conflict of interest}

The authors declare no conflict of interest.

\section{References}

1. Rossignol P, Hernandez AF, Solomon SD, Zannad F. Heart failure drug treatment. Lancet 2019; 393: 1034-44.

2. McMurray JJ, Packer M, Desai AS, et al.; PARADIGM-HF Investigators and Committees. Angiotensin-neprilysin inhibition versus enalapril in heart failure. N Engl J Med 2014; 371: 993-1004.

3. McMurray JJV, Solomon SD, Inzucchi SE, et al.; DAPA-HF Trial Committees and Investigators. Dapagliflozin in patients with heart failure and reduced ejection fraction. N Engl J Med 2019; 381: 1995-2008.

4. Solomon SD, Jhund PS, Claggett BL, et al. Effect of dapagliflozin in patients with $\mathrm{HFrEF}$ treated with sacubitril/valsartan: the DAPA-HF trial. JACC Heart Fail 2020 8: 811-8.

5. Docherty KF, Jhund PS, Anand I, et al. Effect of dapagliflozin on outpatient worsening of patients with heart failure and reduced ejection fraction: a prespecified analysis of DAPA-HF. Circulation 2020; 142: 1623-32.

6. Aimo A, Pateras K, Stamatelopoulos K, et al. Relative efficacy of sacubitril-valsartan, vericiguat, and SGLT2 inhibitors in heart failure with reduced ejection fraction: a systematic review and network meta-analysis. Cardiovasc Drugs Ther 2020 Oct 19. doi: 10.1007/s10557020-07099-2.

7. Seferovic MP, Ponikowski P, Anker S, et al. Clinical practice update on heart failure 2019: pharmacotherapy, procedures, devices and patient management. An expert consensus meeting report of the Heart Failure Association of the European Society of Cardiology. Eur J Heart Fail 2019; 21: 1169-86.

8. Kosiborod MN, Jhund PS, Docherty KF, et al. Effects of dapagliflozin on symptoms, function, and quality of life in patients with heart failure and reduced ejection fraction: results from the DAPA-HF trial. Circulation 2020; 141: 90-9.

9. Wang J, Khoury DS, Thohan V, et al. Global diastolic strain rate for the assessment of left ventricular relaxation and filling pressures. Circulation 2007; 115: 1376-83.

10. Kimura K, Takenaka K, Ebihara A, et al. Speckle tracking global strain rate E/E' predicts LV filling pressure more accurately than traditional tissue Doppler E/E'. Echocardiography 2012; 29: 404-10.
11. Chan YH, Lee HF, Wu LS, et al. Ratio of transmitral early filling velocity to early diastolic strain rate predicts outcomes in patients with systolic heart failure. Eur Heart J Cardiovasc Imaging 2017; 18: 79-85.

12. Chavali V, Tyagi SC, Mishra PK. Predictors and prevention of diabetic cardiomyopathy. Diabetes Metab Syndr Obes 2013; 6: 151-60.

13. Venskutonyte L, Jarnert C, Ryden L, Kjellstrom B. Longitudinal development of left ventricular diastolic function in patients with type 2 diabetes. Diabetes Care 2014; 37: 3092-7.

14. Soga F, Tanaka H, Tatsumi K, et al. Impact of dapagliflozin on left ventricular diastolic function of patients with type 2 diabetic mellitus with chronic heart failure. Cardiovasc Diabetol 2018; 17: 132.

15. Andersen OS, Smiseth OA, Dokainish H, et al. Estimating left ventricular filling pressure by echocardiography. J Am Coll Cardiol 2017; 69: 1937-48.

16. Ponikowski P, Voors AA, Anker SD, et al. 2016 ESC Guidelines for the diagnosis and treatment of acute and chronic heart failure: the Task Force for the diagnosis and treatment of acute and chronic heart failure of the European Society of Cardiology (ESC) Developed with the special contribution of the Heart Failure Association (HFA) of the ESC. Eur Heart J 2016; 37: 2129-200.

17. Ernande L, Bergerot C, Rietzschel ER, et al. Diastolic dysfunction in patients with type 2 diabetes mellitus: is it really the first marker of diabetic cardiomyopathy? J Am Soc Echocardiogr 2011; 24: 1268-75.

18. Oikonomou E, Tousoulis D. Myocardial performance versus exercise tolerance: what matters the most in patients with heart failure? Hellenic J Cardiol 2018; 59: 336-7.

19. Sharma GV, Woods PA, Lambrew CT, et al. Evaluation of a noninvasive system for determining left ventricular filling pressure. Arch Intern Med 2002; 162: 2084-8.

20. Tanaka H, Hirata KI. Potential impact of SGLT2 inhibitors on left ventricular diastolic function in patients with diabetes mellitus. Heart Fail Rev 2018; 23: 439-44.

21. Zinman B, Wanner C, Lachin JM, et al. Empagliflozin, cardiovascular outcomes, and mortality in type 2 diabetes. N Engl J Med 2015; 373: 2117-28.

22. Bailey C, lqbal N, T'joen C, List J. Dapagliflozin monotherapy in drug-naive patients with diabetes: a randomized-controlled trial of low dose range. Diabetes Obes Metab 2012; 14: 951-9.

23. Parikh S, Wilding J, Jabbour S, Hardy E. Dapagliflozin in type 2 diabetes: effectiveness across the spectrum of disease and over time. Int J Clin Pract 2015; 69: 186-98.

24. Filippatos T, Liberopoulos E, Elisaf M. Dapagliflozin in patients with type 2 diabetes mellitus. Ther Adv Endocrinol Metab 2015; 6: 29-41.

25. Wiviott SD, Raz I, Bonaca MP, et al. Dapagliflozin and cardiovascular outcomes in type 2 diabetes. N Engl J Med 2019; 380: 347-57.

26. Papakitsou I, Vougiouklakis G, Elisaf M, Filippatos T. Differential pharmacology and clinical utility of dapagliflozin in type 2 diabetes. Clin Pharmacol 2019; 11: 133-43.

27. Ruanpeng D, Ungprasert P, Sangtian J, et al. Sodium glucose co-transporter 2 (SGLT2) inhibitors and fracture risk in patients with type 2 diabetes mellitus: a meta-analysis. Diabetes Metab Res Rev 2017; 33: 347-56.

28. Kalra S. Sodium glucose co-transporter-2 (SGLT2) inhibitors: a review of their basic and clinical pharmacology. Diabetes Ther 2014; 5: 355-66.

29. Wilcox CS. Antihypertensive and renal mechanisms of SGLT2 (sodium-glucose linked transporter 2) inhibitors. Hypertension 2020; 75: 894-901. 
30. Solomon SD, Verma A, Desai A, et al. Intensive control of hypertension to evaluate efficacy in diastolic dysfunction I. Effect of intensive versus standard blood pressure lowering on diastolic function in patients with uncontrolled hypertension and diastolic dysfunction. Hypertension 2010; 55: 241-8.

31. Haass M, Kitzman DW, Anand IS, et al. Body mass index and adverse cardiovascular outcomes in heart failure patients with preserved ejection fraction: results from the Irbesartan in heart failure with preserved ejection fraction (I-PRESERVE) trial. Circ Heart Fail 2011; 4: 324-31.

32. Ichikawa R, Daimon M, Miyazaki T, et al. Influencing factors on cardiac structure and function beyond glycemic control in patients with type 2 diabetes mellitus. Cardiovasc Diabetol 2013; 12: 38.

33. Ridderstrale M, Andersen KR, Zeller C; Investigators E-RHHSt. Comparison of empagliflozin and glimepiride as add-on to metformin in patients with type 2 diabetes: a 104-week randomised, active-controlled, double-blind, phase 3 trial. Lancet Diabetes Endocrinol 2014; 2: 691-700.

34. Matthaei S, Bowering K, Rohwedder K, et al. Dapagliflozin improves glycemic control and reduces body weight as add-on therapy to metformin plus sulfonylurea: a 24week randomized, double-blind clinical trial. Diabetes Care 2015; 38: 365-72.

35. Soga F, Tanaka H, Tatsumi K, et al. Impact of dapagliflozin on left ventricular diastolic function of patients with type 2 diabetic mellitus with chronic heart failure. Cardiovasc Diabetol 2018; 17: 132.

36. Wang J, Khoury DS, Thohan V, et al. Global diastolic strain rate for the assessment of left ventricular relaxation and filling pressures. Circulation 2007; 115: 1376-83.

37. Kimura K, Takenaka K, Ebihara A, et al. Speckle tracking global strain rate E/E' predicts LV filling pressure more accurately than traditional tissue Doppler E/E'. Echocardiography 2012; 29: 404-410.

38. Chan YH, Lee HF, Wu LS, et al. Ratio of transmitral early filling velocity to early diastolic strain rate predicts outcomes in patients with systolic heart failure. Eur Heart J Cardiovasc Imaging 2017; 18: 79-85. 